Bull. Austral. Math. Soc.

$26 \mathrm{~A} 51,26 \mathrm{D} 15$

VOL. 57 (1998) [377-385]

\title{
QUASI-CONVEX FUNCTIONS AND HADAMARD'S INEQUALITY
}

\section{S.S. Dragomir and C.E.M. Pearce}

\begin{abstract}
Some extensions of quasi-convexity appearing in the literature are explored and relations found between them. Hadamard's inequality is connected tenaciously with convexity and versions of it are shown to hold in our setting. Our theorems extend and unify a number of known results. In particular, we derive a generalised KenyonKlee theorem.
\end{abstract}

\section{INTRODUCTION}

Hadamard's inequality for convex functions has received renewed attention in recent years and a remarkable variety of refinements and generalisations have been found (see, for example, $[1,2,3,4])$. Thus it was shown in [4] that a form of Hadamard's inequality holds for the class $Q(I)$ of Godunova-Levin functions on an interval $I$ of real numbers, that is, the nonnegative functions that satisfy

$$
f(\lambda x+(1-\lambda) y) \leqslant \frac{f(x)}{\lambda}+\frac{f(y)}{1-\lambda}
$$

for all $x, y \in I$ and $\lambda \in(0,1)$.

The class $Q(I)$ contains inter alia all monotone, convex and quasi-convex functions. The class $Q C(I)$ of quasi-convex functions on $I$ is defined as consisting of those functions satisfying

$$
f(\lambda x+(1-\lambda) y) \leqslant \max \{f(x), f(y)\}
$$

for all $x, y \in I$ and $\lambda \in[0,1]$ (see Ponstein [10] and Roberts and Varberg [11, Section 81]).

In [4], the authors introduced the class of real functions of $P$ type, defined as follows.

DEFinition 1.1: Let $I$ be an interval of real numbers. We say that a function $f: I \rightarrow \mathbb{R}$ is of $P$ type, or that $f$ belongs to the class $P(I)$, if $f$ is nonnegative and for all $x, y \in I$ and $\lambda \in[0,1]$ we have

$$
f(\lambda x+(1-\lambda) y) \leqslant f(x)+f(y) .
$$

Received 1st September, 1997

Copyright Clearance Centre, Inc. Serial-fee code: 0004-9729/98 \$A2.00+0.00. 
As seen in [4], $P(I) \subset Q(I)$ but $P(I)$ still contains all monotone, convex and quasiconvex functions. Restricting from $Q$ to $P$ has some importance for applications, as it enables a number of results to be sharpened. In particular, for $f \in P(I) \cap L_{1}[a, b]$ we have the Hadamard-type inequality

$$
f\left(\frac{a+b}{2}\right) \leqslant \frac{2}{b-a} \int_{a}^{b} f(x) d x \leqslant 2(f(a)+f(b))
$$

for $a, b \in I$ and $a<b$. It was also proved in [4] that both inequalities in (1.1) are in a sense best possible.

In this paper we take these ideas rather further and address some generalisations of quasi-convexity appearing in the literature. In Sections 2 and 3 we present inequalities of Hadamard type which hold respectively for Wright-quasi-convex functions and Jensenquasi-convex functions. As consequences we show that both inequalities in (1.1) may be improved for the class of nonnegative quasi-convex functions.

In Section 4, we explore the set-inclusion properties of these classes. We show in particular that quasi-convex functions form a proper subset of the class of Wrightquasi-convex functions and that the latter constitutes a proper subset of the class of Jensen-quasi-convex functions. This mirrors a classical result: it is known that if $C(I)$, $W(I)$ and $J(I)$ are respectively the sets of convex, Wright-convex and Jensen-convex functions on $I$, then

$$
C(I) \subset W(I) \subset J(I)
$$

and each inclusion is proper. This conjecture of Wright [12] was proved by Kenyon [8] and Klee [9].

Hardy, Littlewood and Pólya have remarked [6, Section 3.8] that a convex function is either very regular or very irregular.

Thus if $I$ is open, $f \in J(I)$ and $f$ is not continuous on $I$, then $f$ is unbounded on every open subinterval of $I$ [6, Section 3.18] and $f$ is not measurable on $I$ [6, Section 3.20].

This dichotomy is mitigated somewhat for quasi-convex functions - we shall see in Theorem 4.3 that there are totally discontinuous Jensen quasi-convex functions which are nevertheless bounded - but is still pronounced.

The axiom of choice is invoked implicitly in that we make use of a Hamel basis for the reals (see Hewitt and Stromberg [7, p.18], Hardy, Littlewood and Pólya [6, Section 3.20] and Hamel [5]). In Theorem 4.6 we show that, under the additional constraint of continuity, the classes of quasi-convex, Wright-quasi-convex and Jensen-quasi-convex functions in fact become identical.

In the sequel we assume without further comment that the subset $I \subseteq \mathbb{R}$ refers to an interval. 


\section{HADAMARD'S INEQUALITY FOR $J$-QUASI-CONVEX Functions}

We start with the following definition.

Definition 2.1: The mapping $f: I \rightarrow \mathbb{R}$ is Jensen-or $J$-quasi-convex if

$$
f\left(\frac{x+y}{2}\right) \leqslant \max \{f(x), f(y)\}
$$

for all $x, y \in I$.

Note that the class $J Q C(I)$ of $J$-quasi-convex functions on $I$ contains the class $J(I)$ of $J$-convex functions on $I$, that is, functions satisfying the condition

$$
f\left(\frac{x+y}{2}\right) \leqslant \frac{f(x)+f(y)}{2} \text { for all } x, y \in I .
$$

The following inequality of Hadamard's type holds.

Theorem 2.2. Suppose $a, b \in I \subseteq \mathbb{R}$ and $a<b$. If $f \in J Q C(I) \cap L_{1}[a, b]$, then

$$
f\left(\frac{a+b}{2}\right) \leqslant \frac{1}{b-a} \int_{a}^{b} f(x) d x+I(a, b)
$$

where

$$
I(a, b):=\frac{1}{2} \int_{0}^{1}|f(t a+(1-t) b)-f((1-t) a+t b)| d t
$$

Further, $I(a, b)$ satisfies the inequalities

$$
0 \leqslant I(a, b) \leqslant \frac{1}{b-a} \min \left\{\int_{a}^{b}|f(x)| d x, \frac{1}{\sqrt{2}}\left((b-a) \int_{a}^{b} f^{2}(x) d x-J(a, b)\right)^{1 / 2}\right\},
$$

where

$$
J(a, b):=(b-a)^{2} \int_{0}^{1} f(t a+(1-t) b) f((1-t) a+t b) d t
$$

Proof: Since $f$ is $J$-quasi-convex on $I$, we have for all $x, y \in I$ that

$$
f\left(\frac{x+y}{2}\right) \leqslant \frac{f(x)+f(y)+|f(x)-f(y)|}{2} .
$$

For $t \in[0,1]$, put $x=t a+(1-t) b, y=(1-t) a+t b \in I$. Then

$f\left(\frac{a+b}{2}\right) \leqslant \frac{1}{2}[f(t a+(1-t) b)+f((1-t) a+t b)+|f(t a+(1-t) b)-f((1-t) a+t b)|]$.

Integrating this inequality over $[0,1]$ gives

$$
f\left(\frac{a+b}{2}\right) \leqslant \frac{1}{2}\left[\int_{0}^{1} f(t a+(1-t) b) d t+\int_{0}^{1} f((1-t) a+t b) d t\right]+I(a, b) .
$$

Since

$$
\int_{0}^{1} f(t a+(1-t) b) d t=\int_{0}^{1} f((1-t) a+t b) d t=\frac{1}{b-a} \int_{a}^{b} f(x) d x
$$


(2.1) is proved.

We observe that

$$
\begin{aligned}
0 \leqslant I(a, b) & \leqslant \frac{1}{2}\left[\int_{0}^{1}|f(t a+(1-t) b)| d t+\int_{0}^{1}|f((1-t) a+t b)| d t\right] \\
& =\frac{1}{b-a} \int_{a}^{b}|f(x)| d x
\end{aligned}
$$

On the other hand, by the Cauchy-Buniakowski-Schwarz inequality

$$
\begin{aligned}
\frac{1}{2} \int_{0}^{1} & |f(t a+(1-t) b)-f((1-t) a+t b)| d t \\
& \leqslant \frac{1}{2}\left(\int_{0}^{1}[f(t a+(1-t) b)-f((1-t) a+t b)]^{2} d t\right)^{1 / 2} \\
& =\frac{1}{2}\left[\int_{0}^{1} f^{2}(t a+(1-t) b) d t+\int_{0}^{1} f^{2}((1-t) a+t b) d t\right. \\
& \left.\quad-2 \int_{0}^{1} f(t a+(1-t) b) f((1-t) a+t b) d t\right]^{1 / 2} \\
= & \frac{1}{2}\left[\frac{2}{b-a} \int_{a}^{b} f^{2}(x) d x-2 \int_{0}^{1} f(t a+(1-t) b) f((1-t) a+t b) d t\right]^{1 / 2} \\
& =\frac{\sqrt{2}}{2}\left[\frac{1}{b-a} \int_{a}^{1} f^{2}(x) d x-\int_{0}^{1} f(t a+(1-t) b) f((1-t) a+t b) d t\right]^{1 / 2} .
\end{aligned}
$$

Inequality (2.2) follows.

REMARK 2.3. Suppose $I \subseteq \mathbb{R}$. If $f: I \rightarrow \mathbb{R}$ is quasi-convex and nonnegative, then $f$ is $J$-quasi-convex and thus satisfies

$$
f\left(\frac{a+b}{2}\right) \leqslant \frac{1}{b-a} \int_{a}^{b} f(x) d x+I(a, b) \leqslant \frac{2}{b-a} \int_{a}^{b} f(x) d x
$$

which improves the first inequality in (1.1) for quasi-convex functions.

\section{HADAMARD'S INEQUALITY FOR $W$-QUASI-CONVEX FUNCTIONS}

Wright introduced an interesting class of functions in [12].

We say $f: I \rightarrow \mathbb{R}$ is a Wright-convex function on $I \subseteq \mathbb{R}$ if, for each $y>x$ and $\delta>0$ with $y+\delta, x \in I$ we have

$$
f(x+\delta)-f(x) \leqslant f(y+\delta)-f(y) .
$$

The following characterisation holds for $W$-convex functions (see also [4]).

Propos ITION 3.1. Suppose $I \subseteq \mathbb{R}$. Then the following statements are equivalent for a function $f: I \rightarrow \mathbb{R}$.

(i) $f$ is $W$-convex on $I$; 
(ii) for all $a, b \in I$ and $t \in[0,1]$, we have the inequality

$$
f(t a+(1-t) b)+f((1-t) a+t b) \leqslant f(a)+f(b) .
$$

PROOF: For "(i) $\Rightarrow$ (ii)", let $a, b \in I$ and $t \in[0,1]$. First suppose $a<b$.

If $f$ is $W$-convex on $I$, then for all $y>x$ and $\delta>0$ with $y+\delta, x \in I$ we have (W). Choose $x=a, y=t a+(1-t) b>0$ and $\delta:=b-(t a+(1-t) b)>0$. Then $x+\delta=(1-t) a+t b, y+\delta=b$ and thus by (W) we get

$$
f((1-t) a+t b)-f(a) \leqslant f(b)-f(t a+(1-t) b),
$$

whence we have (3.1).

The proof is similar for the case $a>b$.

For "(ii) $\Rightarrow$ (i)", let $y>x$ and $\delta>0$ with $y+\delta, x \in I$. In (3.1) choose $a=x, b>a$ and $t \in[0,1]$ with $t a+(1-t) b=y$ and $b-(t a+(1-t) b)=\delta$. We have $y+\delta=b \in I$, $x \in I$ and $x+\delta=(1-t) a+t b$. From (3.1) we derive

$$
f(x)+f(y+\delta) \geqslant f(y)+f(x+\delta)
$$

which shows that the map is $W$-convex on $I$.

The equivalence motivates the introduction of the following class of functions.

Definition 3.2: For $I \subseteq \mathbb{R}$, the mapping $f: I \rightarrow \mathbb{R}$ is Wright-quasi-convex if, for all $x, y \in I$ and $t \in[0,1]$, one has the inequality

$(W Q C)$

or equivalently

$$
\frac{1}{2}[f(t x+(1-t) y)+f((1-t) x+t y)] \leqslant \max \{f(x), f(y)\}
$$

$$
\frac{1}{2}[f(y)+f(x+\delta)] \leqslant \max \{f(x), f(y+\delta)\}
$$

for every $x, y+\delta \in I$ with $x<y$ and $\delta>0$.

We show that the following inequality of Hadamard type holds.

THEOREM 3.3. Let $f: I \rightarrow \mathbb{R}$ be a $W$-quasi-convex map on $I$ and suppose $a, b \in I \subseteq \mathbb{R}$ with $a<b$ and $f \in L_{1}[a, b]$. Then we have the inequality

$$
\frac{1}{b-a} \int_{a}^{b} f(x) d x \leqslant \max \{f(a), f(b)\} .
$$

Proof: For all $t \in[0,1]$ we have

$$
\frac{1}{2}[f(t a+(1-t) b)+f((1-t) a+t b)] \leqslant \max \{f(a), f(b)\} .
$$

On integrating this inequality over $[0,1]$ and using $(2.3)$ we obtain the desired inequality.

REMARK 3.4. If $f$ is quasi-convex and nonnegative, then

$$
\frac{1}{b-a} \int_{a}^{b} f(x) d x \leqslant \max \{f(a), f(b)\} \leqslant f(a)+f(b),
$$

which improves the second inequality in (1.1) for quasi-convex and nonnegative functions. 


\section{Set-Inclusion Properties}

We begin by introducing, by analogy with some of the other classes considered in this paper, the notion of a quasi-monotone function.

Definition 4.1: For $I \subseteq \mathbb{R}$, the mapping $f: I \rightarrow \mathbb{R}$ is quasi-monotone if it is either monotone on $I=[c, d]$ or monotone nonincreasing on a proper subinterval $\left[c, c^{\prime}\right] \subset I$ and monotone nondecreasing on $\left[c^{\prime}, d\right]$.

The class $Q M(I)$ of quasi-monotone functions on $I$ provides an immediate characterisation of quasi-convex functions.

Propos ITION 4.2. Suppose $I \subseteq \mathbb{R}$. Then the following statements are equivalent for a function $f: I \rightarrow \mathbb{R}$.

(a) $f \in Q M(I)$;

(b) on any compact subinterval of $I, f$ achieves a supremum at an end point;

(c) $f \in Q C(I)$.

Proof: That (a) implies (b) is immediate from the definition of quasi-monotonicity. For the reverse implication, suppose if possible that (b) holds but $f \notin Q M(I)$. Then there must exist points $x, y, z \in I$ with $x<y<z$ and $f(y)>\max [f(x), f(z)]$, contradicting (b) for the subinterval $[x, z]$. The equivalence of (b) and (c) is just the definition of quasi-convexity.

The following inclusion results hold.

THEOREM 4.3. Let $W Q C(I)$ denote the class of Wright-quasi-convex functions on $I \subseteq \mathbb{R}$. Then

$$
Q C(I) \subset W Q C(I) \subset J Q C(I)
$$

Both inclusions are proper.

Proof: Let $f \in Q C(I)$. Then for all $x, y \in I$ and $t \in[0,1]$ we have

$$
f(t x+(1-t) y) \leqslant \max \{f(x), f(y)\}, \quad f((1-t) x+t y) \leqslant \max \{f(x), f(y)\}
$$

which gives by addition that

$$
\frac{1}{2}[f(t x+(1-t) y)+f((1-t) x+t y)] \leqslant \max \{f(x), f(y)\}
$$

for all $x, y \in I$ and $t \in[0,1]$, that is, $f \in W Q C(I)$. The second inclusion is obvious on choosing $t=1 / 2$ in (4.2).

Let $H$ be a Hamel basis over the rationals. Then each real number $u$ has a unique representation

$$
u=\sum_{h \in H} r_{u, h} h
$$


in which only finitely many of the coefficients $r_{u, h}$ are nonzero. Define a mapping $f: I \rightarrow$ $\mathbb{R}$ by

$$
f(u)=\sum_{u \in H} r_{u, h}
$$

Then

$$
\begin{aligned}
\frac{1}{2}[f(y)+f(x+\delta)] & =\frac{1}{2}\left[\sum_{h} r_{y, h}+\sum_{h}\left(r_{x, h}+r_{\delta, h}\right)\right] \\
& =\frac{1}{2}\left[\sum_{h} r_{x, h}+\sum_{h}\left(r_{y, h}+r_{\delta, h}\right)\right] \\
& \leqslant \max \left\{\sum_{h} r_{x, h}, \sum_{h}\left(r_{y, h}+r_{\delta, h}\right)\right\} \\
& =\max \{f(x), f(y+\delta)\}
\end{aligned}
$$

so that $f \in W Q C(I)$.

We now show that $H$ can be selected so that $f \notin Q C(I)$. Choose $x=0, y=1, \lambda=$ $1-(1 / \pi)$ and take $2,(1 / \pi) \in H$. Then

$$
f(\lambda x+(1-\lambda) y)=f(1 / \pi)=1>\max \{0,1 / 2\}=\max \{f(x), f(y)\} .
$$

Hence $f \notin Q C(I)$.

For the second inclusion in (4.1), consider the Dirichlet map $f: I \rightarrow \mathbb{R}$ defined by

$$
f(u)= \begin{cases}1 & \text { for } u \text { irrational } \\ 0 & \text { for } u \text { rational. }\end{cases}
$$

If $x$ and $y$ are both rational, then so is $(x+y) / 2$, so that in this case

$$
f\left(\frac{x+y}{2}\right)=\max \{f(x), f(y)\} .
$$

If one of $x, y$ is rational and the other irrational, then $(x+y) / 2$ is irrational and so again (4.3) holds. If both $x$ and $y$ are irrational, then $\max \{f(x), f(y)\}=1$, so that

$$
f\left(\frac{x+y}{2}\right) \leqslant \max \{f(x), f(y)\} .
$$

Hence $f \in J Q C(I)$. However if $x$ and $y$ are distinct rationals, there are uncountably many values of $t \in(0,1)$ for which $t x+(1-t) y$ and $(1-t) x+t y$ are both irrational. For each such $t$

$$
\frac{1}{2}[f(t x+(1-t) y)+f((1-t) x+t y)]>\max \{f(x), f(y)\}
$$

so that $f \notin W Q C(I)$. Hence $W Q C(I)$ is a proper subset of $J Q C(I)$. 
THEOREM 4.4. We have the inclusions

$$
W(I) \subset W Q C(I), \quad C(I) \subset Q C(I), \quad J(I) \subset J Q C(I) .
$$

Each inclusion is proper.

Proof: By Lemma 3.1, we have for $f \in W(I)$ that

$$
\frac{1}{2}[f(t a+(1-t) b)+f((1-t) a+t b)] \leqslant \frac{f(a)+f(b)}{2}
$$

for all $a, b \in I$ and $t \in[0,1]$.

Since

$$
\frac{f(a)+f(b)}{2} \leqslant \max \{f(a), f(b)\} \text { for all } a, b \in I,
$$

the inequality in Definition 3.2 is satisfied, that is, $f \in W Q C(I)$ and the first inclusion is thus proved. Similar proofs hold for the other two.

By (1.2) and (4.1), for each inclusion to be proper it is sufficient that there should exist a function $f$ with $f \in Q C(I)$ but $f \notin J(I)$. Clearly any strictly concave monotone function suffices.

REMARK 4.5. In view of the result of the foregoing theorem, the fact that there are functions in $Q C(I)$ which are not in $J(I)$ makes it tempting to try to concatenate the set inclusions (1.2) and (4.1). However, no result of this sort appears to exist without the imposition of further assumptions. Thus, for example, by the use of a Hamel basis, solutions to (3.1) may be constructed which are unbounded on every subinterval, whereas all members of $Q C(I)$ are bounded on every finite interval. Hence it is not the case that $W(I) \subset Q C(I)$.

We now show that the three notions of quasi-convex functions in Theorem $4.3 \mathrm{col}$ lapse into one under the additional constraint of continuity. We denote by $Q M_{0}(I)$ the class of quasi-monotone functions under this constraint, with similar notation for the other classes involved in Theorems 4.3 and 4.4.

THEOREM 4.6. For a given interval $I \subseteq \mathbb{R}$,

$$
Q C_{0}(I)=W Q C_{0}(I)=J Q C_{0}(I) .
$$

Proof: The proofs of the basic inclusion results of Theorem 4.3 do not involve continuity, so that

$$
Q C_{0}(I) \subset W Q C_{0}(I) \subset J Q C_{0}(I) .
$$

For the same reason, by Proposition 4.2 we have $Q M_{0}(I)=Q C_{0}(I)$. Hence it suffices to prove that $J Q C_{0}(I) \subset Q M_{0}(I)$. We proceed by reductio ad absurdum.

Suppose, if possible, that $f \in J Q C_{0}(I)$ but that $f \notin Q M_{0}(I)$. Then there must exist points $x, y, z \in I$ with $x<z<y$ and $f(z)>f(x)=f(y)$. Let $|y-x|=d$. By 
continuity there exists an open interval $I_{0} \subset[x, y]$ of length $d_{0}(>0)$ with $z \in I_{0}$ and $f$ strictly exceeding $f(x)$ on $I_{0}$. Since $f \in J Q C(I)$, we have $(x+y) / 2 \notin I_{0}$, so that $I_{0}$ is properly contained in either $(x,(x+y) / 2]$ or $[(x+y) / 2, y)$.

Invoking continuity again, there must be, according to which of these two cases holds, either a point $x^{\prime} \in(x,(x+y) / 2]$ with $f\left(x^{\prime}\right)=f(x)$ and $I_{0} \subset\left(x, x^{\prime}\right)$ or a point $y^{\prime} \in[(x+y) / 2, y)$ with $f\left(y^{\prime}\right)=f(y)$ and $I_{0} \subset\left(y^{\prime}, y\right)$. Call this interval $\left(x^{\prime}, y^{\prime}\right)$. Then $f\left(x^{\prime}\right)=f\left(y^{\prime}\right),\left|y^{\prime}-x^{\prime}\right| \leqslant d / 2$ and $I_{0} \in\left(x^{\prime}, y^{\prime}\right)$.

The previous argument may be repeated to show that there exist $x^{\prime \prime}, y^{\prime \prime}$ with $f\left(x^{\prime \prime}\right)=$ $f\left(y^{\prime \prime}\right),\left|y^{\prime \prime}-x^{\prime \prime}\right| \leqslant d / 4$ and $I_{0} \subset\left(x^{\prime \prime}, y^{\prime \prime}\right)$. A continuation of this process yields that $d / 2^{n}>d_{0}$ for all $n \geqslant 1$, which is impossible.

REMARK 4.7. Theorem 4.4 does not extend in this way. Thus, for example, if $f$ is continuous, strictly concave and monotone, we have $f \in Q M_{0}$ but $f \notin W_{0}$.

\section{REFERENCES}

[1] S.S. Dragomir, 'Two mappings on connection to Hadamard's inequality', J. Math. Anal. Appl. 167 (1992), 49-56.

[2] S.S. Dragomir, 'On Hadamard's inequalities for convex functions', Mat. Balkanica 6 (1992), 215-222.

[3] S.S. Dragomir, J.E. Pečarić and J. Sándor, 'A note on the Jensen-Hadamard inequality', Anal. Num. Théor. Approx. 19 (1990), 29-34.

[4] S.S. Dragomir, J.E. Pečarić and L.E. Persson, 'Some inequalities of Hadamard type', Soochow J. Math. 21 (1995), 335-341.

[5] G. Hamel, 'Eine Basis aller Zahlen und die unstetigen Lösungen der Funktionalgleichung: $f(x+y)=f(x)+f(y)^{\prime}$, Math. Ann. 60 (1905), 459-462.

[6] G.H. Hardy, J.E. Littlewood and G. Pólya, Inequalities (Cambridge University Press, Cambridge, 1934).

[7] E. Hewitt and K. Stromberg, Real and abstract analysis (Springer-Verlag, Berlin, Heidelberg, New York, 1965).

[8] H. Kenyon, 'Note on convex functions', Amer. Math. Monthly 63 (1956), 107.

[9] V.L. Klee, 'Solution of a problem of E.M. Wright on convex functions', Amer. Math. Monthly 63 (1956), 106-107.

[10] J. Ponstein, 'Seven types of convexity', SIAM Review 9 (1967), 115-119.

[11] A.W. Roberts and D.E. Varberg, Convex functions (Academic Press, New York, 1973).

[12] E.M. Wright, 'An inequality for convex functions', Amer. Math. Monthly 61 (1954), 620-622.

Applied Mathematics Department

University of Transkei

UNITRA, Private Bag XI

Umtata 5100

South Africa
Department of Applied Mathematics The University of Adelaide Adelaide SA 5005

Australia 